\title{
The impact of smoking on severity and outcome in patients with COVID-19 infection in Mosul city
}

\author{
Mohammad Alsaaty ${ }^{1}$, Abdullah alyyouzbaki ${ }^{1}$, and wael thanoon ${ }^{1}$ \\ ${ }^{1}$ University of Mosul
}

June 30, 2021

\begin{abstract}
Background: The novel coronavirus ( SARS CoV 19) is responsible for the pandemic infection after starting in 2019 in Wuhan city /China, several small studies were done since then to determine the effects of smoking on the severity, outcome \& mortality of patients with COVID-19 but the results were inconclusive, this study aims to assess the impact of active smoking on severity and outcome in patients with COVID-19 infection in Mosul city/Iraq. Methods: A prospective cohort study was conducted on 160 patients ( 80 patients were active smokers \& 80 non-smoker patients) who have been diagnosed with COVID-19 infection by using real-time PCR, the study was done at Ibn-Sina teaching hospital in Mosul city/Iraq from May 2020 until December 2020. A detailed history was taken from the patients, full clinical examination \& blood tests were done. The following parameters were recorded \& compared between the 2 groups: severity of symptoms, inflammatory markers, D dimer, liver function test (LFT), oxygen use, hospitalization \& outcome. Results: the symptoms of COVID-19 were more severe in the non smokers group $(34.4 \%)$ compared to the smokers $(25 \%) \&$ it was statistically significant (p-value $=0.016)$, the inflammatory markers were also higher in the non-smokers $(31.9 \%)$ compared to the smokers $(20.0 \%)$ ( p-value $=0.011)$, D dimer \& LFT were also higher in the non-smoker group ( $\mathrm{p}$-value $=0.01 \& 0.008$ respectively). There was no statistical difference regarding oxygen use, hospitalization, ICU admission, mortality, or post-recovery complications. Conclusion: severe symptomatic COVID-19 infection was more common in the non-smoker group, the inflammatory markers, D-dimer \& LFT were higher in the nonsmokers compared to the smokers. No statistical difference was found regarding O2 use, hospitalization, ICU admission, mortality \& residual complications.
\end{abstract}

\section{Hosted file}

smoking and covid.docx available at https://authorea.com/users/422819/articles/528458-theimpact-of-smoking-on-severity-and-outcome-in-patients-with-covid-19-infection-in-mosulcity 